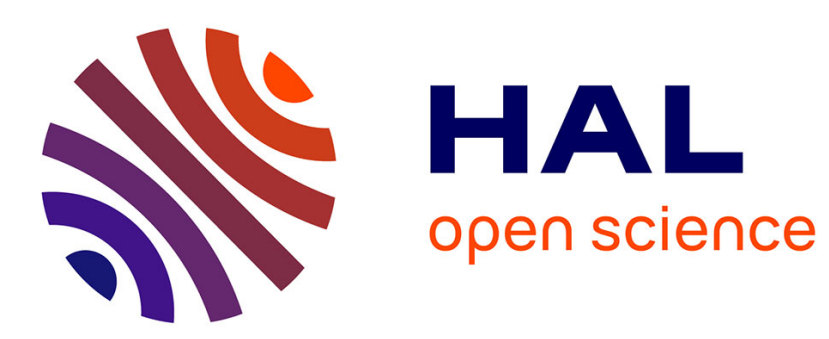

\title{
Bézier Representation of Trim Curves
}

Dieter Lasser, Georges-Pierre Bonneau

\section{- To cite this version:}

Dieter Lasser, Georges-Pierre Bonneau. Bézier Representation of Trim Curves. Dagstuhl Seminar on

Geometric Modelling, 1993, Dagstuhl, Germany. pp.1-18. hal-01708592

\section{HAL Id: hal-01708592 \\ https://hal.inria.fr/hal-01708592}

Submitted on 13 Feb 2018

HAL is a multi-disciplinary open access archive for the deposit and dissemination of scientific research documents, whether they are published or not. The documents may come from teaching and research institutions in France or abroad, or from public or private research centers.
L'archive ouverte pluridisciplinaire HAL, est destinée au dépôt et à la diffusion de documents scientifiques de niveau recherche, publiés ou non, émanant des établissements d'enseignement et de recherche français ou étrangers, des laboratoires publics ou privés. 


\section{Interner Bericht}

\section{Bézier Representation \\ of}

Trim Curves

$235 / 93$

Dieter Lasser, Georges-Pierre Bonneau

Fachbereich Informatik

Universität Kaiserslautern

Kaiserslautern

\section{Fachbereich Informatik}

Universität Kaiserslautern - Postfach 3049 - D-67653 Kaiserslautern 


\section{Bézier Representation of Trim Curves}

$235 / 93$

Dieter Lasser, Georges-Pierre Bonneau

Fachbereich Informatik

Universität Kaiserslautern

Kaiserslautern

Herausgeber: AG Graphische Datenverarbeitung und Computergeometrie Leiter: Prof. Dr. H. Hagen

Kaiserslautern, im Dezember 1993 


\title{
Bézier Representation of Trim Curves
}

\author{
Dieter Lasser, Georges-Pierre Bonneau \\ Computer Science \\ University of Kaiserslautern \\ Germany
}

\begin{abstract}
The composition of Bézier curves and tensor product Bézier surfaces, polynomial as well as rational, is applied to exactly and explicitely represent trim curves of tensor product Bézier surfaces. Trimming curves are assumed to be defined as Bézier curves in surface parameter domain. A Bézier spline approximation of lower polynomial degree is built up as well which is based on the exact trim curve representation in coordinate space.
\end{abstract}

Keywords. Bézier representation, trim curves, curves on surfaces, composition, approximation, free form deformation

\section{Introduction}

Very few three-dimensional objects are representable by a single (free form) surface patch. Generally surfaces have to be glued together smoothly as in a patch work to form spline surfaces, and (spline) surfaces have to be processed further within CAD/CAM solid modelling systems. Some of these interrogation techniques, such as intersecting and blending, trim surfaces to an area bounded by border curves (see Figure 1) commonly refered to as trim curves. In context of blending they are also called contact or link curves. This is to highlight the fact that in blending, trim curves are curves of contact along which primary surface and blend surface are linked together (see Figure 2).

Literature on the subject of trim curves and trimmed surfaces can be grouped into three categories. First, there is the solid modelling, second, the computer graphic, and third, the CAGD oriented literatuie. 


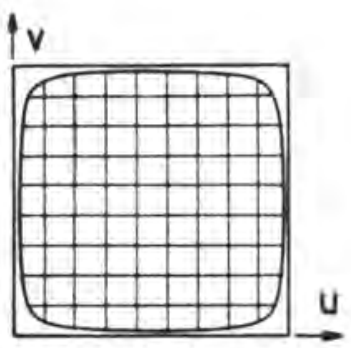

dome surface

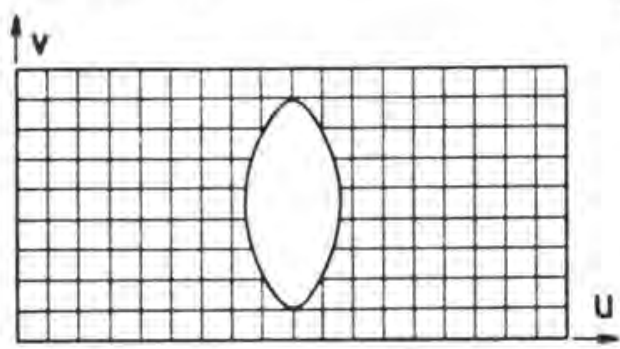

shield surface

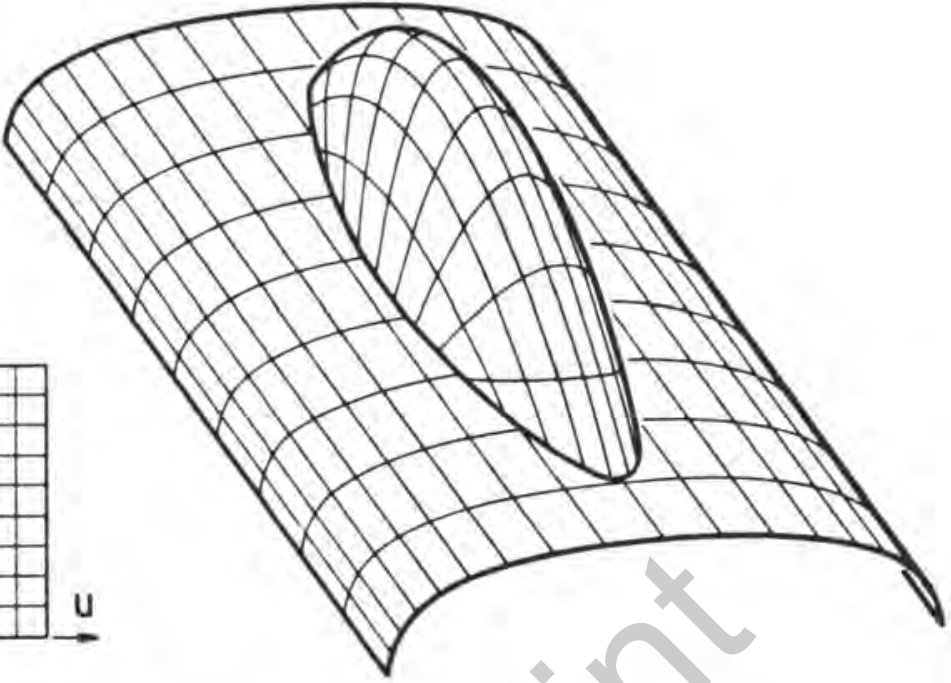

Figure 1. Surface-surface intersection creates trimmed surfaces
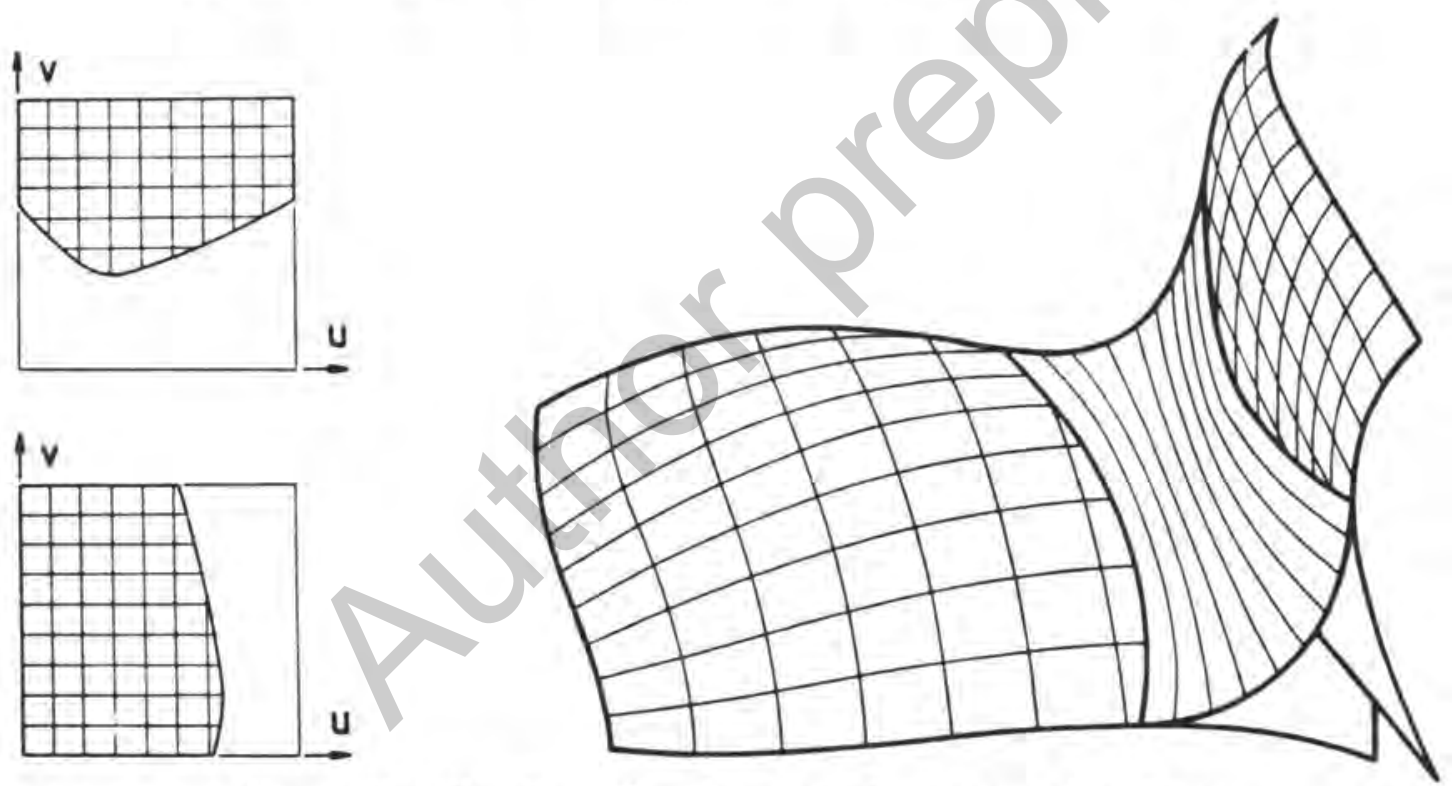

Figure 2. Blending of surfaces creates trimmed surfaces

Undoubtedly, solid modelling is the area with most contributions. Issues discussed are the ones most important for Boolean set operation and for boundary evaluation: First, the intersection problem of trimmed surfaces and of sculptured solids, i.e. solids with a free form outer (trimmed) surface, and second, set membership classification, which classifies a point as being interior to, on the boundary of, or exterior to a set [Cas 87, 89], [Cro 87], [Faro 87]. Engineering analysis interrogation algorithms for solids bounded by trimmed surfaces, i.e. computation of surface area, volume, center of gravity, moments of inertia and other mechanical or mass properties are subject of [Faro 87] and [Cas 92]. [She 92] triangulates trimmed surfaces for the purpose of data exchange between CAD systems and a stereolithography apparatus to generate a solid hard copy directly from a 3D CAD model. The faceting algorithm described in [She 92] is suitable for rendering of parametrically defined surfaces. 
Rendering is also the main issue of the computer graphic oriented literature on trimmed surfaces. Although, very different methods are applied: [Roc89] creates a faceting of trimmed rational tensor product surfaces. Facets are lighted, smooth shaded and zbuffered using standard 3D polygon rendering techniques. [Sha 88] performs a scan line based rendering of trimmed NURBS surfaces using adaptive forward differencing together with a Hermite shading function approximation along surface curves. [Nis 90] renders trimmed rational tensor product Bézier surfaces using the ray tracing methology, While [Roc 89] and [Sha 88] have to calculate intersection points of isoparametric lines and trim curves to actually perform trimming, [Nis 90 ] realizes trimming by point classification, i.e. by determining if a point on a patch lies inside or outside a trimmed region.

In CAGD contributions on trimmed surfaces concentrate on two issues: data exchange between CAD systems and blending of surfaces. In blending, determination of trim curves is one of the crucial points. It can be done interactively or automatically, in coordinate space or in parameter space [Bar 89], [Fil 89], [Cho 89], [Har 90], [Kla 92], [Kop 91], [Peg 90]. Data exchange is subject of [Hos90], describing an approximate conversion of NURBS surfaces trimmed by NURBS curves by lower degree B-spline representations, and of [Vri 91, 92], exactly converting a polynomial tensor product Bézier surface trimmed by polynomial Bézier curves into a composite Bézier surface. The later algorithm is based on subdividing the trimmed parameter space region of a surface in planar three- and four-gonal regions, and on the substitution of a bilinear Coons representation of these parameter space areas into the surface representation.

Most algorithms assume trim curves $\mathbf{K}(t)$ to be given in polynomial or rational representation in parameter space of surfaces $\mathbf{F}(u, v): \mathbb{R}^{2} \rightarrow \mathbb{R}^{3}$, i.e. $\mathbf{K}(t)=(u(t), v(t)): \mathbb{R} \rightarrow \mathbb{R}^{2}$, even if they have been calculated approximatively or in discrete points only, by surface intersection or projection, for example. Further trim curve processing then usually involves one or more approximation procedures at different levels of processing.

While it is well understood, that the mathematical nature of trimming is functional composition, $\mathbf{F}(\mathbf{K}(t))$, see Figure 3, evaluation of $\mathbf{F}(\mathbf{K}(t))$ is done throughout the literature pointwise and up to very recently no exact closed form representation of $\mathbf{F}(\mathbf{K}(t))$ had been given, except for the triviale case of monomial representations of $\mathbf{K}(t)$ and of $\mathbf{F}(u, v)$,

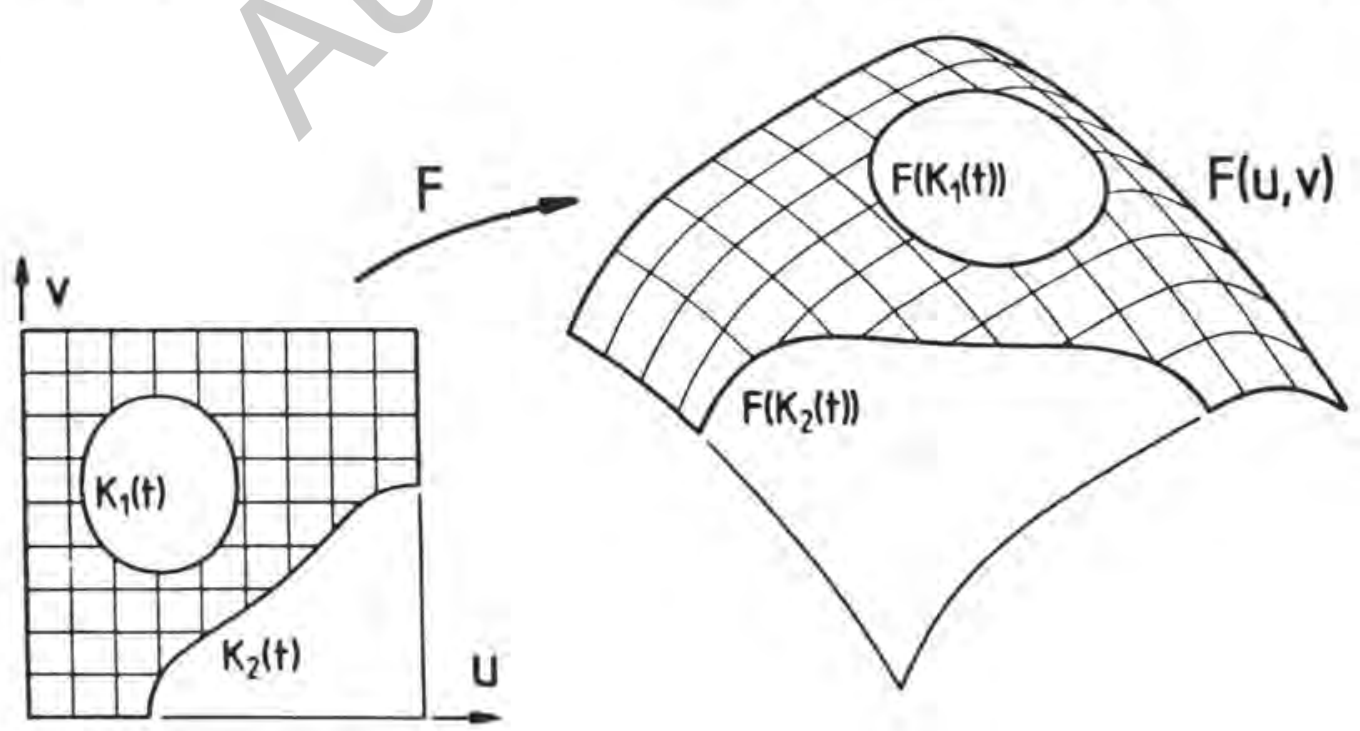

Figure 3. The mathematical nature of trimming is composition 
which can be found in [Bez 78] already (in context of free form deformation application). [Vri 91, 92] also gives an explicit and exact representation of compositions, in context of trim curves and trimmed tensor product surfaces. Though the work is primarily done for Bézier representations, [Vri 91,92] unnecessarily performs conversions at several stages of the algorithm which makes the method less accurate, [Faro 91]. Composition especially is done in the monomial bases only, which requires conversion to and from this bases.

To have an exact and explicite Bézier representation of compositions of Bézier representations would be of great value for many CAD/CAM, computer graphics and CAGD applications. T. DeRose, [DeR 88], has been the first who actually performed functional composition in case of simplicial Bézier representations. DeRose also pointed out a whole selection of applications of functional composition such as evaluation, subdivision, (nonlinear) reparametrization and geometric continuity. A more interesting example of application might be given by the idea of curve and surface modeling in the sense of free form deformation. A second, very important application of functional composition concerns the subject of curves on surfaces, e.g. trimming of surfaces by surface curves, with applications in blending and in solid modeling, i.e. trimmed surfaces. [Elb 92] looks at Bézier curves and tensor product Bézier surfaces, both polynomial and rational. But there are no explicit Bézier-like equations given for $\mathbf{F}(\mathbf{K}(t))$ and furthermore, Table 6.1 of [Elb 92] listing polynomial degrees of $\mathbf{F}(\mathbf{K}(t))$ is erroneous. [Las 91] provided exact Bézier-representations of $\mathbf{F}(\mathbf{K}(t))$ for polynomial and rational Bézier curves and surfaces, multivariate composition is treated as well. [DeR 93] deals with the same topic, but is highlighting the blossoming nature of algorithms much stronger than [Las 91] does.

In this paper we apply (and discuss a special case of) the theoretical results given in [Las 91] to the problem of exactly representing curves on surfaces, here trim curves, in Bézier form. An approximative representation is given as well. The paper is structured as follows: Section II reviews definitions of Bézier curves and surfaces, In Sections III and V Theorem 1 (polynomial case) and Theorem 2 (rational case) are given which are fundamental for the exact representation of Bézier curves on tensor product Bézier surfaces. Sections IV and VI apply Theorem 1 and Theorem 2 to exactly and explicitly represent trim curves of surfaces. Section VII notes one more application of functional composition and of theorems given in Sections III and V, planar curve design via free form deformation. Section VIII is concerned with the task of approximating trim curves by lower degree polynomials.

\section{Bézier Representations}

A planar Bézier curve $\mathbf{K}(t)$ of degree $N$ in $t$ is defined by

$$
\mathbf{K}(t)=\sum_{l=0}^{N} \mathbf{K}_{l} B_{I}^{N}(t), \quad t \in[0,1]
$$

where $\mathbf{K}_{I} \in \mathbb{R}^{2}$ and

$$
B_{I}^{N}(t)=\left(\begin{array}{c}
N \\
I
\end{array}\right) t^{I}(1-t)^{N-I}
$$

are the (ordinary) Bernstein polynomials of degree $N$ in $t$. 
A tensor product Bézier surface $\mathbf{F}(u, v)$ - briefly TPB-surface - of degree $(l, m)$ in $(u, v)$ is defined by

$$
\mathbf{F}(u, v)=\sum_{i=0}^{l} \sum_{j=0}^{m} \mathbf{F}_{i, j} B_{i}^{l}(u) B_{j}^{m}(v), \quad u, v \in[0,1],
$$

where $\mathbf{F}_{i, j} \in \mathbb{R}^{3}$ and with Bernstein polynomials $B_{i}^{l}(u)$ and $B_{j}^{m}(v)$. By reason of the tensor product definition algorithms in $u$ and in $v$ commute, and the result is independent of the order.

A planar rational Bézier curve $\mathbf{K}(t)$ of degree $N$ in $t$ is defined by

$$
\mathbf{K}(t)=\frac{\sum_{I=0}^{N} \beta_{I} \mathbf{K}_{I} B_{I}^{N}(t)}{\sum_{I=0}^{N} \beta_{I} B_{I}^{N}(t)}, \quad t \in[0,1] .
$$

and a rational tensor product Bézier surfaces $\mathbf{F}(u, v)$ briefly rational TPBsurface - of degree $(l, m)$ in $(u, v)$ is defined by

$$
\mathbf{F}(u, v)=\frac{\sum_{i=0}^{l} \sum_{j=0}^{m} \omega_{i, j} \mathbf{F}_{i, j} B_{i}^{l}(u) B_{j}^{m}(v)}{\sum_{i=0}^{l} \sum_{j=0}^{m} \omega_{i, j} B_{i}^{l}(u) B_{j}^{m}(v)}, u, v \in[0,1] .
$$

Coefficients $\mathbf{K}_{I}$ and $\mathbf{F}_{i, j}$ are called Bézier points. They form in their natural ordering, given by their subscripts, the vertices of the Bézier polygon and of the Bézier net. Scalars $\beta_{I} \in \mathbb{R}$ and $\omega_{i, j} \in \mathbb{R}$ are called weights. Positive weights result in curves and surfaces which have all the properties and algorithms which we do have for polynomial representations.

The Bézier description is a very powerful tool because the expansion in terms of Bernstein polynomials yields, first, a numerically very stable behavior of all algorithms. And, second, a geometric meaning of Bézier points (and weights). For an extensive coverage of properties of Bernstein polynomials and Bézier representations see e,g. [Far 90], [Hos 92].

\section{Composition of Polynomial Curves and Surfaces}

Theorem 1 describes the composition $\mathbf{F}(t)=\mathbf{F}(\mathbf{K}(t))=\mathbf{F}(u(t), v(t))$ of a planar Bézier curve $\mathbf{K}(t)$ and a TPB-surface $\mathbf{F}(u, v)$. There are several problems in curve and surface modeling pointed out by DeRose [DeR 88] that can be solved using functional composition. Examples are evaluation, subdivision, (nonlinear) reparametrization and geometric continuity of Bézier representations. Two more examples of practical interest are curve and surface modeling via free-form deformation and the description of curves on surfaces. The later can be thought of as trim curves with applications in blending as well as in connection with trimmed surfaces as the occur in solid modeling. In that context Theorem 1 is fundamental for the exact representation of trim curves. 
Theorem 1. Let $\mathbf{K}(t)=(u(t), v(t)): \mathbb{R} \rightarrow \mathbb{R}^{2}$ be a planar polynomial Bézier curve of degree $N$, (1), with Bézier points $\mathbf{K}_{I}=\left(u_{I}, v_{I}\right)$, and let $\mathbf{F}(u, v)=(x(u, v), y(u, v), z(u, v))$ : $\mathbb{R}^{2} \rightarrow \mathbb{R}^{3}$, be a polynomial TPB-surface of degree $(l, m),(2)$, with Bézier points $\mathbf{F}_{i, j}=$ $\left(x_{i, j}, y_{i, j}, z_{i, j}\right) . \mathbf{F}(t)=\mathbf{F}(\mathbf{K}(t))=\mathbf{F}(u(t), v(t))$ is polynomial and can be represented as Bézier curve of degree $r N$, where $r=l+m$. We have

$$
\mathbf{F}(t)=\mathbf{F}(\mathbf{K}(t))=\sum_{R=0}^{r N} \mathbf{B}_{R} B_{R}^{r N}(t),
$$

with Bézier points

$$
\mathbf{B}_{R}=\sum_{|\mathbf{I}|=R} C_{R}^{l, m}(N, \mathbf{I}) \mathbf{F}_{0,0}^{l, m}\left(u_{\mathbf{I}^{u}}^{l}, v_{\mathbf{I}^{\mathrm{v}}}^{m}\right),
$$

and constants

$$
C_{R}^{l, m}(N, \mathbf{I})=\frac{\prod_{Q^{u}=1}^{l}\left(\begin{array}{c}
N \\
I_{Q^{*}}^{u}
\end{array}\right) \prod_{Q^{v}=1}^{m}\left(\begin{array}{c}
N \\
I_{Q^{*}}^{v}
\end{array}\right)}{\left(\begin{array}{c}
r N \\
R
\end{array}\right)}
$$

Proof of Theorem 1 is done analogously to [DeR 88] and has been given in detail in [Las 91] (cf. [DeR 93]).

$\sum_{|\mathbf{I}|=R}$ has the meaning of summation over all $\mathbf{I}=\left(\mathbf{I}^{u}, \mathbf{I}^{u}\right)$, where $\mathbf{I}^{u}=\left(I_{1}^{u}, \ldots, I_{l}^{u}\right)$, $\mathbf{I}^{v}=\left(I_{1}^{v}, \ldots, I_{m}^{v}\right)$ and where $0 \leq I_{1}^{u}, \ldots, I_{l}^{u} \leq N$ and $0 \leq I_{1}^{v}, \ldots, I_{m}^{v} \leq N$ and $|\mathbf{I}|=\left|\mathbf{I}^{u}\right|+\left|\mathbf{I}^{v}\right|=I_{1}^{u}+\ldots+I_{l}^{u}+I_{1}^{u}+\ldots+I_{m}^{\nu}=R$.

Note, construction points $\mathbf{F}_{0,0}^{l, m}\left(u_{\mathrm{I}^{\mathrm{u}}}^{l}, v_{\mathrm{I}^{\mathrm{v}}}^{\mathrm{m}}\right)$ arise in the calculation of the polar form of $\mathbf{F}(u, v)$. They can be computed recursively using the de Casteljau's construction, i.e. for the $u$ parameter direction by

$$
\mathbf{F}_{i, j}^{i+\alpha, j+\beta}\left(u_{I^{u}}^{\alpha}, v_{I^{v}}^{\beta}\right)=\left(1-u_{I_{\alpha}^{u}}\right) \mathbf{F}_{i, j}^{i+\alpha-1, j+\beta}\left(u_{I^{u}}^{\alpha-1}, v_{I^{v}}^{\beta}\right)+u_{I_{\alpha}^{u}} \mathbf{F}_{i+1, j}^{i+\alpha, j+\beta}\left(u_{I^{u}}^{\alpha-1}, v_{I^{v}}^{\beta}\right),
$$

and for the $v$ parameter direction by

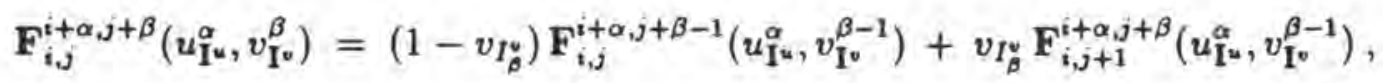

where $\mathbf{F}_{i, j}^{i, j} \equiv \mathbf{F}_{i, j}$. The argument $\left(u_{\mathbf{I}^{u}}^{o}, v_{\mathbf{I}^{v}}^{\beta}\right)$ has the meaning that $\mathbf{F}_{i, j}^{i+\alpha, j+\beta}$ has to be calculated by performing $\alpha$ de Casteljau constructions in $u$ direction for the $u$ parameter values given by the indices $\mathbf{I}^{u}=\left(I_{1}^{u}, \ldots, I_{\alpha}^{u}\right)$, i.e. for the parameter values $u_{I_{1}^{\prime}}, \ldots, u_{I_{\alpha}^{*}}$ and $\beta$ de Casteljau constructions in $v$ direction for the $v$ parameter values given by the indices $I^{v}=\left(I_{1}^{v}, \ldots, I_{\beta}^{v}\right)$, i.e. for the parameter values $v_{I_{1}^{v}}, \ldots, v_{I_{\beta}^{v}}$. Calculations for different parameter values commute, and the order of performed calculations does not effect the final result.

The special case $N=1$ deserves some extra attention: While it is well known, that for a TPB-surface of degree $(l, m)$ isoparameter lines map to Bézier curves of degree $l$ or $m$, respectively, which can be calculated by applying the de Casteljau (subdivision) algorithm, Theorem 1 generalizes this statement in the sense that now we know, lines 
of parameter space in general position map to Bézier curves of degree $l+m .^{1}$ Actually, Theorem 1 does not really differentiate between isoparametric lines and lines in general position. Theorem 1 represents both of them as Bézier curves of degree $l+m$. This sounds like Theorem 1 is wrong. Indeed, the only way it can work is that,

Statement 1. In case of isoparametric lines Theorem 1 yields degree raised Bézier curves.

Proof. To prove Statement 1 , we go with the following strategy: First, we assume $\mathbf{K}(t)$ be isoparametric and specialize Theorem 1 to $N=1$, second, we show that $\mathbf{F}(t)$ is degree raised, and third, we perform degree reduction and compare the result with the Bézier representation of isoparametric lines, calculated using the de Casteljau (subdivision) algorithm:

First, we specialize Theorem 1 to $N=1$ and assume (because of the tensor product structure of $\mathbf{F}(u, v))$ w.l.o.g. $\mathbf{K}(t), t \in[0,1]$, being given as part $u \in\left[u_{0}, u_{1}\right] \subseteq[0,1]$ of a $u$ parameter line, i.e. $v_{0}=v_{1} \equiv v_{*}$. (calculations and arguments are analogous in case of a $v$ parameter line $\left.u_{0}=u_{1} \equiv u_{*}\right)$. This should result in a parametric curve $\mathbf{F}(\mathbf{K}(t))=\mathbf{F}\left(u, v_{-}\right), u \in\left[u_{0}, u_{1}\right]$, of degree $l$ in $u$ :

Because of $N=1$, i.e. $I_{Q^{u}}^{u} \in\{0,1\}$ and $I_{Q^{v}}^{\nu} \in\{0,1\}$, (5) becomes

and (4) simplifies to

$$
C_{R}^{l, m}(N, \mathbf{I})=\frac{1}{\left(\begin{array}{c}
l+m \\
R
\end{array}\right)}
$$

$$
\mathbf{B}_{R}=\frac{1}{\left(\begin{array}{c}
1+m \\
R
\end{array}\right)} \sum_{|\mathbf{I}|=R} \mathbf{F}_{\mathbf{0}, 0}^{l, m}\left(u_{\mathbf{I}^{u}}^{l}, v_{\mathbf{I}}^{m}\right) .
$$

Because de Casteljau constructions commute, and the polar form of $\mathbf{F}(u, v)$ is symmetric w.r.t. permutations of the argument, i.e. $\mathbf{F}_{0,0}^{l, m}\left(u_{\mathbf{I}^{u}}^{l}, v_{\mathbf{I}^{v}}^{m}\right)$ is so, the $\operatorname{sum} \sum_{|\mathbf{I}|=R} \mathbf{F}_{0,0}^{l, m}\left(u_{\mathbf{I}^{v}}^{l}, v_{\mathbf{I}^{*}}^{m}\right)$ is in the case of $v_{0}=v_{1} \equiv v$. equivalent to $\sum_{a=0}^{R}\left(\begin{array}{l}l \\ a\end{array}\right)\left(\begin{array}{c}m \\ R-a\end{array}\right) \mathbf{F}_{a}$, using $\left(\begin{array}{l}n \\ k\end{array}\right) \equiv 0$ for $k<0$ and for $k>n$, and where $\mathbf{F}_{a}$ stands for $\mathbf{F}_{0,0}^{l, m}\left(u_{\mathrm{I}^{u}}^{l}, v_{*}^{m}\right)$ with $a$ being the number of indices $I_{Q^{u}}^{u}$ of $\mathbf{I}^{u}$ of value 1, i.e. $a=\left|\mathbf{I}^{u}\right| \leq l$. Thus far, the representation of $\mathbf{F}(\mathbf{K}(t))$ according to Theorem 1 simplifies to

$$
\mathbf{F}(t)=\mathbf{F}(\mathbf{K}(t))=\sum_{R=0}^{l+m} \mathbf{B}_{R} B_{R}^{l+m}(t),
$$

with

$$
\mathbf{B}_{R}=\sum_{a=0}^{R} \frac{\left(\begin{array}{c}
l \\
a
\end{array}\right)\left(\begin{array}{c}
m \\
R-a
\end{array}\right)}{\left(\begin{array}{c}
1+m \\
R
\end{array}\right)} \mathbf{F}_{a} .
$$

Now, $\mathbf{F}(\mathbf{K}(t))$ is supposed to be of degree $l$ instead of degree $l+m$. To prove the degree $l$ of $\mathbf{F}(\mathbf{K}(t))$ we calculate forward differences

$$
\Delta^{k} \mathbf{B}_{R}=\sum_{j=0}^{k}(-1)^{j}\left(\begin{array}{l}
k \\
j
\end{array}\right) \mathbf{B}_{R+k-j},
$$

\footnotetext{
${ }^{1}$ This is, by the way, in contrast to the situation for triangle Bézier surfaces. In case of a triangle Bézier surface of degree $n$ isoparametric lines as well as lines of parameter space in general position map to Bézier curves of degree $n$.
} 
with $\mathbf{B}_{R+k-j}$ given by (10), and then prove validity of

$$
\bigwedge_{i=1, \ldots, m} \bigwedge_{R=0, \ldots, m-i} \Delta^{l+i} \mathbf{B}_{R}=0 .
$$

Bézier points of the degree $l$ representation finally result by degree reduction of (9), (10) from degree $l+m$ to degree $l$.

The last two steps, proof of (11) and degree reduction, are quite cumbersome. That is why we prefere to go a short-cut and are doing both steps at the same time:

First, we note, that $(10)$ is equivalent to

$$
\mathbf{B}_{R}=\sum_{\substack{a=R-m \\
a \geq 0}}^{R} \frac{\left(\begin{array}{l}
l \\
a
\end{array}\right)\left(\begin{array}{c}
m \\
R-a
\end{array}\right)}{\left(\begin{array}{c}
l+m \\
R
\end{array}\right)} \mathbf{F}_{\alpha},
$$

and second, we realize, that (12) is the degree raising formula for raising the polynomial degree of a Bézier curve of degree $l$ defined by Bézier points $\mathbf{F}_{\mathrm{a}}$ from $l$ to $l+m$. This completes the proof, because $\mathbf{F}_{a}$ stands for $\mathbf{F}_{0,0}^{l, m}\left(u_{\mathrm{I} u}^{l}, v_{\cdot}^{m}\right)$, which means that $\mathbf{F}_{a}$ has been calculated using the de Casteljau algorithm exactly the precise number of times and for the correct parameter values.

Remark 1. Bézier splines can be treated easily, too. First, we determine in the domain space of $\mathbf{F}(u, v)$ the intersections of the spline curve $\mathbf{K}(t)$ and the patch edges of spline surface $\mathbf{F}(u, v)$ and split $\mathbf{K}(t)$ at these intersection points by adding new knots applying de Casteljau's subdivision algorithm. For all segments of $\mathbf{F}(u, v)$, all spline curve segments of $\mathbf{K}(t)$ situated in the $(u, v)$ domain of the specific spline segment of $\mathbf{F}(u, v)$ can now be treated directly by Theorem 1 . If $\mathbf{K}(t)$ is $C^{a}$-continuous in $t=t^{*}$, and $\mathbf{F}(u, v)$ is $C^{b}$-continuous in the corresponding point $\mathbf{K}(t)=\mathbf{K}\left(t^{*}\right)$ of $(u, v)$ space, $\mathbf{F}(\mathbf{K}(t))$ is $C^{e}$-continuous in $\mathbf{F}\left(\mathbf{K}\left(t^{*}\right)\right.$, with $e=\min \{a, b\}$, [Las 91].

\section{Polynomial Trim Curves and Surfaces}

Applying Theorem 1, curves on surfaces (e.g. trim curves) can be represented exactly, provided both curve as well as surface are of polynomial nature and, in that case, w.l.o.g. are given in Bézier representation.

Assuming $\mathbf{K}(t)$ not being degree raised, we first check, in the event of $N=1$, by comparing $\mathbf{K}_{0}$ with $\mathbf{K}_{1}$, if $\mathbf{K}(t)$ is isoparametric. If so, $\mathbf{F}(\mathbf{K}(t))$ is not of degree $l+m$ and, we do not have to calculate Bézier points $\mathbf{B}_{R}$ of $\mathbf{F}(\mathbf{K}(t))$ using equations (6) and (7). Following Statement $1, \mathbf{F}(\mathbf{K}(t))$ is of degree $l$ or $m$, respectively, instead of degree $l+m$, and Bézier points $\mathbf{B}_{R}$ are defined by $\mathbf{B}_{R} \equiv \mathbf{F}_{0,0}^{l, m}\left(u_{I^{*}}^{l}, v_{*}^{m}\right)$, where $\left|\mathbf{I}^{u}\right|=R$, in case of $\mathbf{K}(t)$ being $u$ parameter line $v_{0}=v_{1} \equiv v_{*}$, or by $\mathbf{B}_{R} \equiv \mathbf{F}_{0,0}^{l, m}\left(u_{-}^{l}, v_{\mathbf{I} v}^{m}\right)$, where $\left|\mathbf{I}^{v}\right|=R$, in case of $\mathbf{K}(t)$ being $v$ parameter line $u_{0}=u_{1} \equiv u_{*}$, respectively. Otherwise, $\mathbf{F}(\mathbf{K}(t))$ is of degree $N(l+m)$, and it's Bézier representation is given by Theorem 1 .

Figures 3-6 illustrate Theorem 1 and Statement 1 for the examples of trimming Bézier (spline) curves defined in the domains of TPB-surfaces. In case of Figure 3 a closed cubic $C^{1}$ subspline and a quartic curve are mapped on a TPB-surface of degree $(2,4)$. 

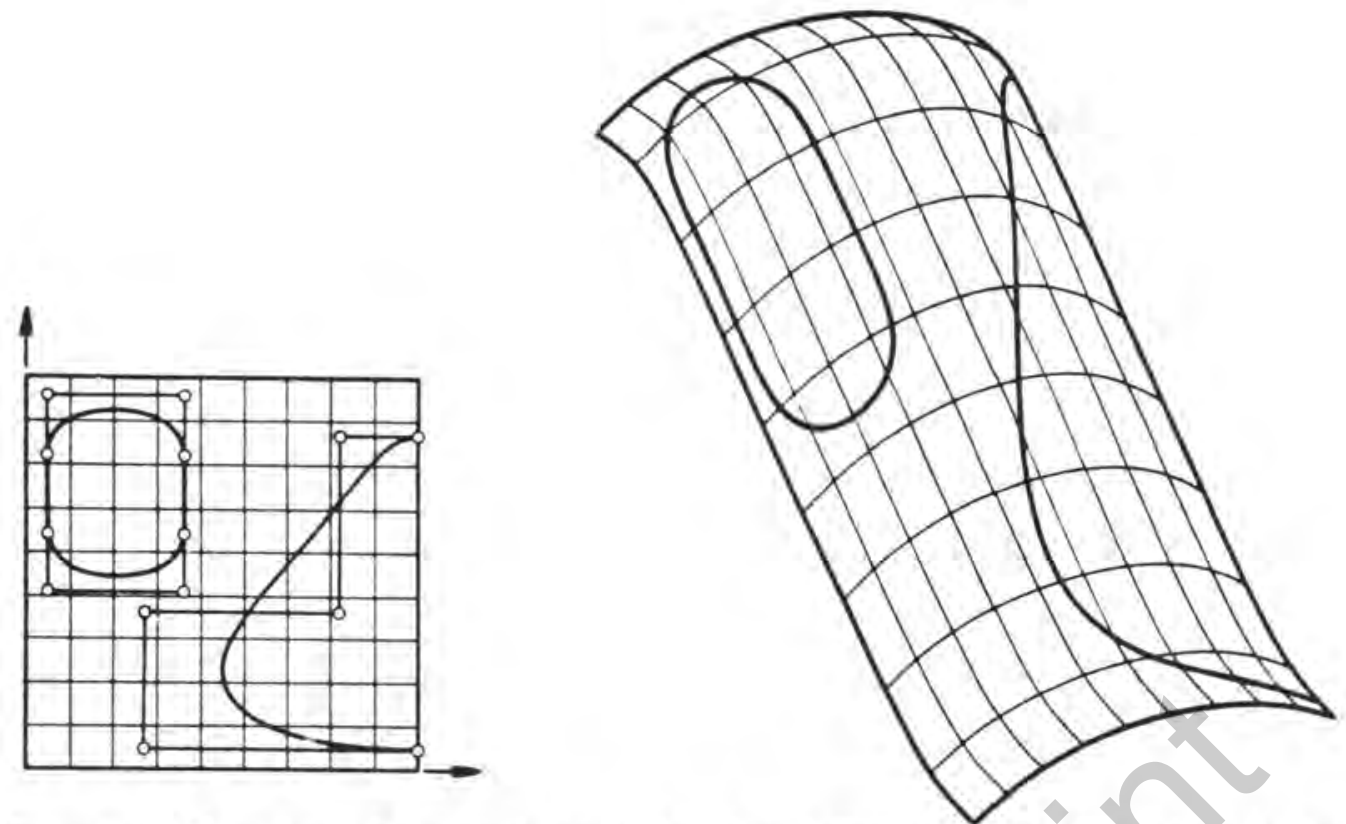

Figure 4. Mapping of a quintic and of a closed $G C^{1}$ linear/cubic trimming curve on a TPB-surface of degree $(5,3)$
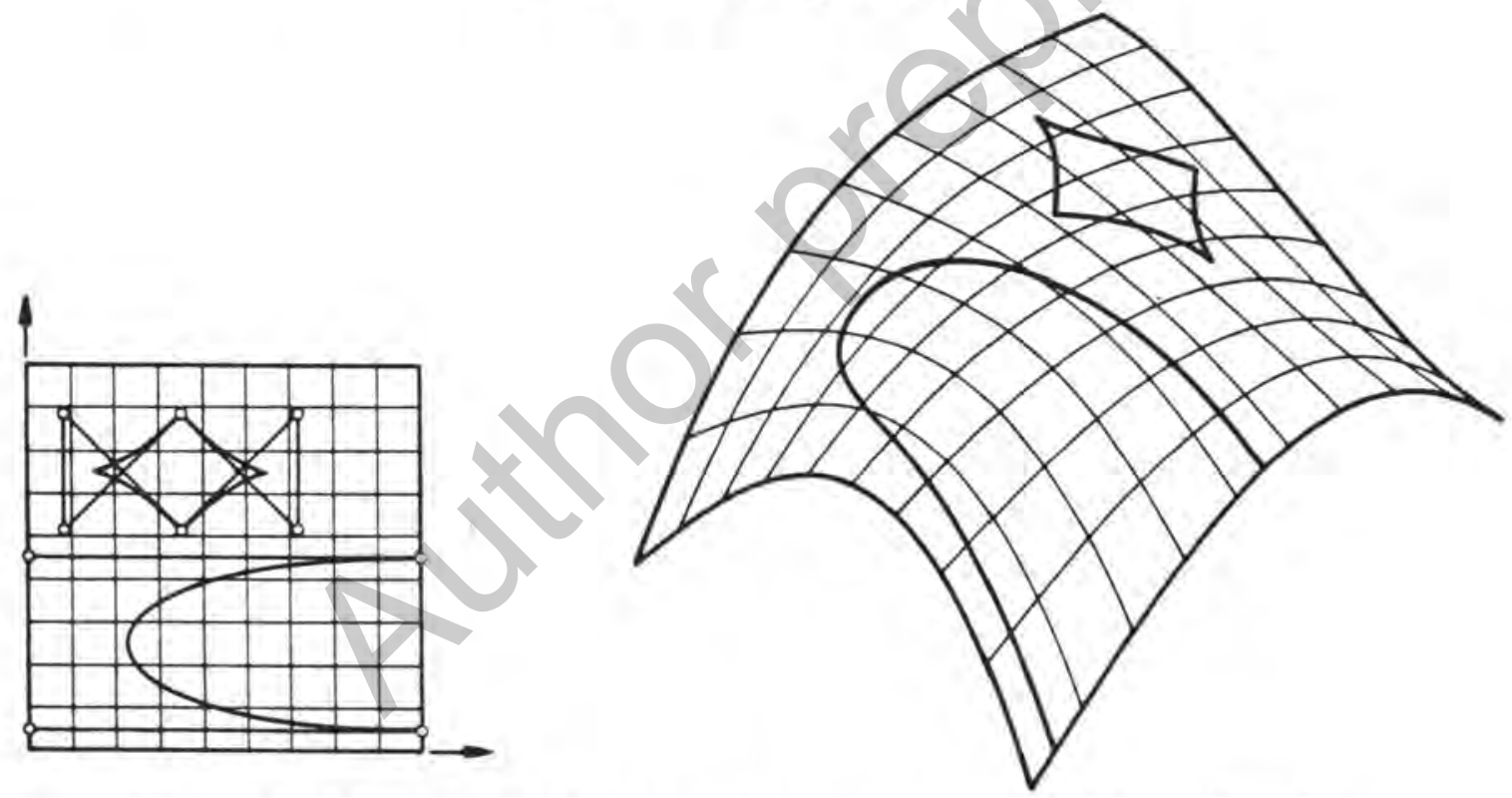

Figure 5. Mapping of cubic trimming curves on a bicubic TPB-surface

\section{Composition of Rational Curves and Surfaces}

Theorem 2 describes the composition of a planar rational Bézier curve of degree $N, \mathbf{K}(t)$, defined in domain space of a rational TPB-surface of degree $(l, m), \mathbf{F}(u, v)$.

Theorem 2. Let $\mathbf{K}(t)=(u(t), v(t)): \mathbb{R} \rightarrow \mathbb{R}^{2}$ be a planar rational Bézier curve of degree $N$, (3), with Bézier points $\mathbf{K}_{I}=\left(u_{I}, v_{I}\right)$ and weights $\beta_{I}$. And let $\mathbf{F}(u, v)=$ $(x(u, v), y(u, v), z(u, v)): \mathbb{R}^{2} \rightarrow \mathbb{R}^{3}$, be a rational TPB-surface of degree $(l, m),(4)$, with 

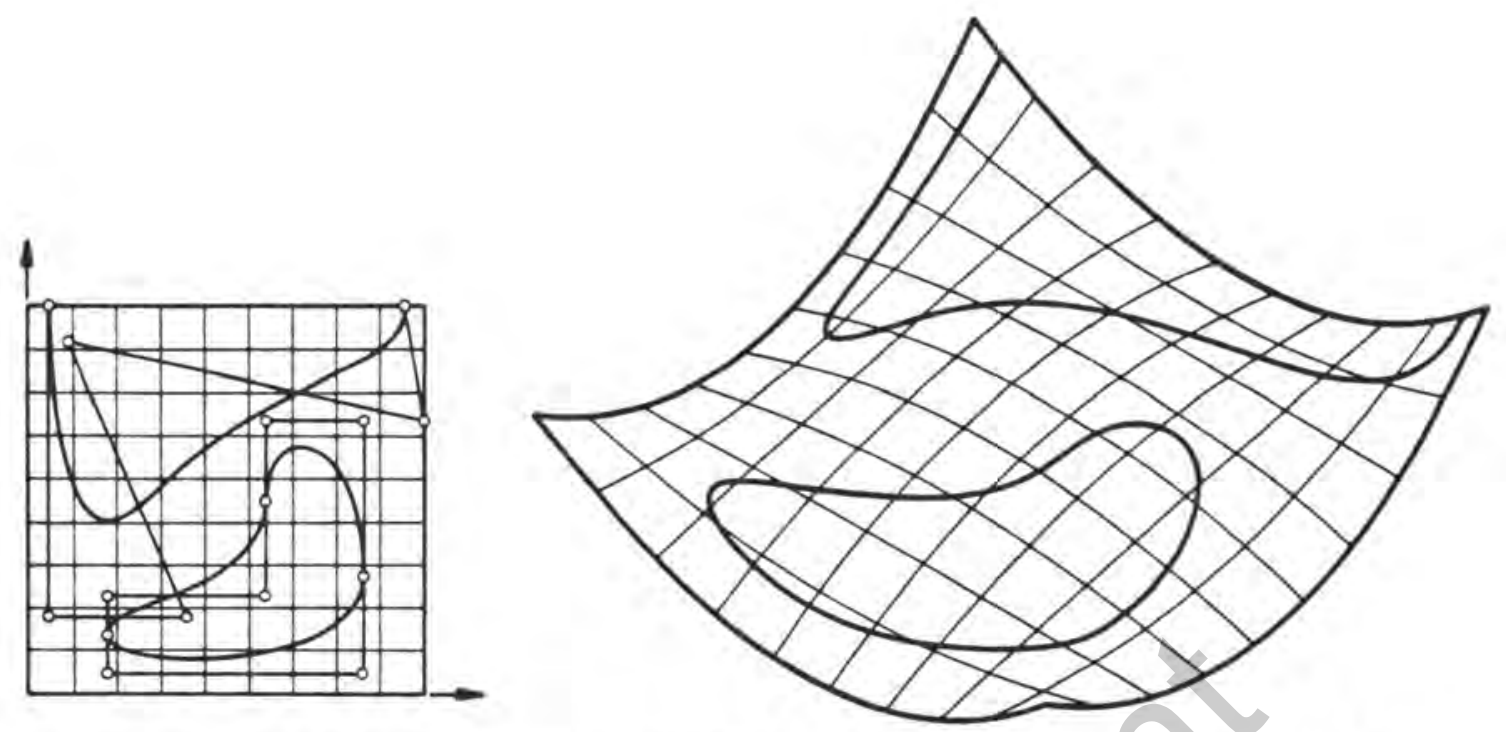

Figure 6. Mapping of a closed cubic $G C^{1}$ subspline and of a quintic trimming curve on a biquadratic TPB-surface

Bézier points $\mathbf{F}_{i, j}=\left(x_{i, j}, y_{i, j}, z_{i, j}\right)$ and weights $\omega_{i, j} . \mathbf{F}(t)=\mathbf{F}(\mathbf{K}(t))=\mathbf{F}(u(t), v(t))$ is rational and can be represented as rational Bézier curve of degree $r N$, where $r=l+m$. We have

$$
\mathbf{F}(t)=\mathbf{F}(\mathbf{K}(t))=\frac{\sum_{R=0}^{r N} \Omega_{R} B_{R} B_{R}^{r N}(t)}{\sum_{R=0}^{r N} \Omega_{R} B_{R}^{r N}(t)} .
$$

Weights are given by

$$
\Omega_{R}=\sum_{|\mathbf{I}|=R} B_{R}^{l, m}(N, \mathbf{I}) \omega_{0,0}^{l, m}\left(u_{\mathbf{I}^{\mathrm{u}}}^{l}, v_{\mathbf{I}^{\mathrm{v}}}^{m}\right),
$$

Bézier points are given by $\mathbf{B}_{R}=\frac{\Omega_{R} \mathbf{B}_{R}}{\Omega_{R}}$, where

$$
\Omega_{R} \mathbf{B}_{R}=\sum_{|\mathbf{I}|=R} B_{R}^{l, m}(N, \mathbf{I}) \omega_{0,0}^{l, m}\left(u_{\mathbf{I}^{u}}^{l}, v_{\mathbf{I}^{v}}^{m}\right) \mathbf{F}_{0,0}^{l, m}\left(u_{\mathbf{I}^{u}}^{l}, v_{\mathbf{I}^{v}}^{m}\right),
$$

and with constants

$$
B_{R}^{l, m}(N, \mathbf{I})=\frac{\prod_{Q^{v}=1}^{l} \beta_{I_{Q^{u}}^{u}}\left(\begin{array}{c}
N \\
I_{Q^{u}}^{u}
\end{array}\right) \prod_{Q^{v}=1}^{m} \beta_{I_{Q^{v}}^{v}}\left(\begin{array}{c}
N \\
I_{Q^{v}}^{v}
\end{array}\right)}{\left(\begin{array}{c}
r N \\
R
\end{array}\right)} .
$$

Proof of Theorem 2 is essentially like the one of Theorem 1 with the difference, that rational representations are now involved. Proof of Theorem 2 has been given in [Las 91]. 
$\sum_{|\mathbf{I}|=R}$ and $\mathbf{I}=\left(\mathbf{I}^{u}, \mathbf{I}^{\nu}\right)$ have the same meaning as in Theorem 1. $\omega_{0,0}^{l, m} \mathbf{F}_{0,0}^{l, m}$ and $\omega_{0,0}^{l, m}$ are defined recursively by de Casteljau's construction, analogously to Theorem 1.

Theorem 2 maps straight lines of parameter space to rational Bézier curves of degree $l+m$. We have to show consistency of Theorem 2 with the fact that isoparametric lines actually map to rational Bézier curves of degree $l$ or $m$, respectively. Therefore, we formulate

Statement 2. In case of isoparametric lines Theorem 2 yields degree raised rational Bézier curves.

Proof. Statement 2 can be proven exactly the same way Statement 1 has been: Let $N=1$ and $\mathbf{K}(t), t \in[0,1]$, being given as part $u \in\left[u_{0}, u_{1}\right] \subseteq[0,1]$ of a $u$ parameter line, i.e. $v_{0}=v_{1} \equiv v_{*}$. (calculations and arguments are analogous in case of a $v$ parameter line $u_{0}=u_{1} \equiv u_{*}$ ). W.l.o.g. $\beta_{0}$ and $\beta_{N}$ can be chosen equal to one, $\beta_{0}=\beta_{N}=1$. Now, because of $N=1$, these are the only weights defining $\mathbf{K}(t)$ and furthermore, we have $I_{Q^{u}}^{u} \in\{0,1\}$ and $I_{Q^{v}}^{v} \in\{0,1\}$. Equation (16) which defines $B_{R}^{l, m}(N, \mathbf{I})$ therefore simplifies to (8) and equations (14) and (15) become (cf. Statement 1)

$$
\begin{aligned}
\Omega_{R} & =\sum_{\substack{a=R-m \\
a \geq 0}}^{R} \frac{\left(\begin{array}{l}
l \\
a
\end{array}\right)\left(\begin{array}{c}
m \\
R-a
\end{array}\right)}{\left(\begin{array}{c}
l+m \\
R
\end{array}\right)} \omega_{a}, \\
\Omega_{R} \mathbf{B}_{R} & =\sum_{\substack{a=R-m \\
a \geq 0}}^{R} \frac{\left(\begin{array}{l}
l \\
a
\end{array}\right)\left(\begin{array}{c}
m \\
R-a
\end{array}\right)}{\left(\begin{array}{c}
l+m \\
R
\end{array}\right)} \omega_{a} \mathbf{F}_{a},
\end{aligned}
$$

where $\omega_{a}$ and $\omega_{a} \mathbf{F}_{a}$ are short for $\omega_{0,0}^{l, m}\left(u_{\mathrm{I}^{\mu}}^{l}, v_{*}^{m}\right)$ and for $\omega_{0,0}^{l, m}\left(u_{\mathrm{I}^{*}}^{l}, v_{*}^{m}\right) \mathbf{F}_{0,0}^{l, m}\left(u_{\mathrm{I}^{u}}^{l}, v_{*}^{m}\right)$ with $a=\left|\mathbf{I}^{u}\right| \leq l$. Realizing that $\left(\omega_{a} \mathbf{F}_{a}, \omega_{a}\right)$ as well as $\left(\Omega_{R} \mathbf{B}_{R}, \Omega_{R}\right)$ are $4 \mathrm{D}$-Bézier points of a 4D homogeneous coordinate representation of rational Bézier curves we see, that (17) and (18) are degree raising formulas for rational Bézier curves for raising the degree from $l$ to $l+m$, and we are done.

Remark 2. Rational Bézier splines can be treated as described in Remark 1.

Remark 3. Theorem 2 includes special cases $\mathbf{K}(t)$ and $\mathbf{F}(u, v)$ both being polynomial, $\mathbf{K}(t)$ being polynomial and $\mathbf{F}(u, v)$ being rational, and $\mathbf{K}(t)$ being polynomial and $\mathbf{F}(u, v)$ being rational. In the first case statement of Theorem 1 results. Special cases two and three both yield rational curves, [Las 91].

\section{Rational Trim Curves and Surfaces}

According to Theorem 2 and Statement 2 we go with the following strategy (assuming $\mathbf{K}(t)$ not being degree raised):

If $N=1$ and $\mathbf{K}_{0}$ and $\mathbf{K}_{1}$ are isoparametric, $\mathbf{F}(\mathbf{K}(t))$ is of degree $l$ defined by weights $\Omega_{R} \equiv$ $\omega_{0,0}^{l, m}\left(u_{\mathrm{I} v}^{l}, v_{*}^{m}\right)$ and by Bézier points $\mathbf{B}_{R}$ defined via $\Omega_{R} \mathbf{B}_{R} \equiv \omega_{0,0}^{l, m}\left(u_{\mathrm{Iv}}^{l}, v_{*}^{m}\right) \mathbf{F}_{0,0}^{l, m}\left(u_{\mathrm{I} v}^{l}, v_{*}^{m}\right)$, where $\left|\mathbf{I}^{u}\right|=R$, in case of $\mathbf{K}(t)$ being $u$ parameter line $v_{0}=v_{1} \equiv v_{*}$, and $\mathbf{F}(\mathbf{K}(t))$ is of degree $m$ defined by weights $\Omega_{R} \equiv \omega_{0,0}^{l, m}\left(u_{*}^{l}, v_{\mathbf{I} v}^{m}\right)$ and by Bézier points $\mathbf{B}_{R}$ defined via 
$\Omega_{R} \mathbf{B}_{R} \equiv \omega_{0,0}^{l, m}\left(u_{*}^{l}, v_{\mathbf{I}^{*}}^{m}\right) \mathbf{F}_{0,0}^{l, m}\left(u_{*}^{l}, v_{\mathbf{I}^{v}}^{m}\right)$, where $\left|\mathbf{I}^{v}\right|=R$, in case of $\mathbf{K}(t)$ being $v$ parameter line $u_{0}=u_{1} \equiv u_{\text {. }}$. Otherwise $\mathbf{F}(\mathbf{K}(t))$ is of degree $N(l+m)$ with Bézier representation according to Theorem 2 .

Figures 7 and 8 illustrate Theorem 2 and Statement 2 for the examples of rational trimming Bézier (spline) curves defined in the domains of rational TPB-surfaces.
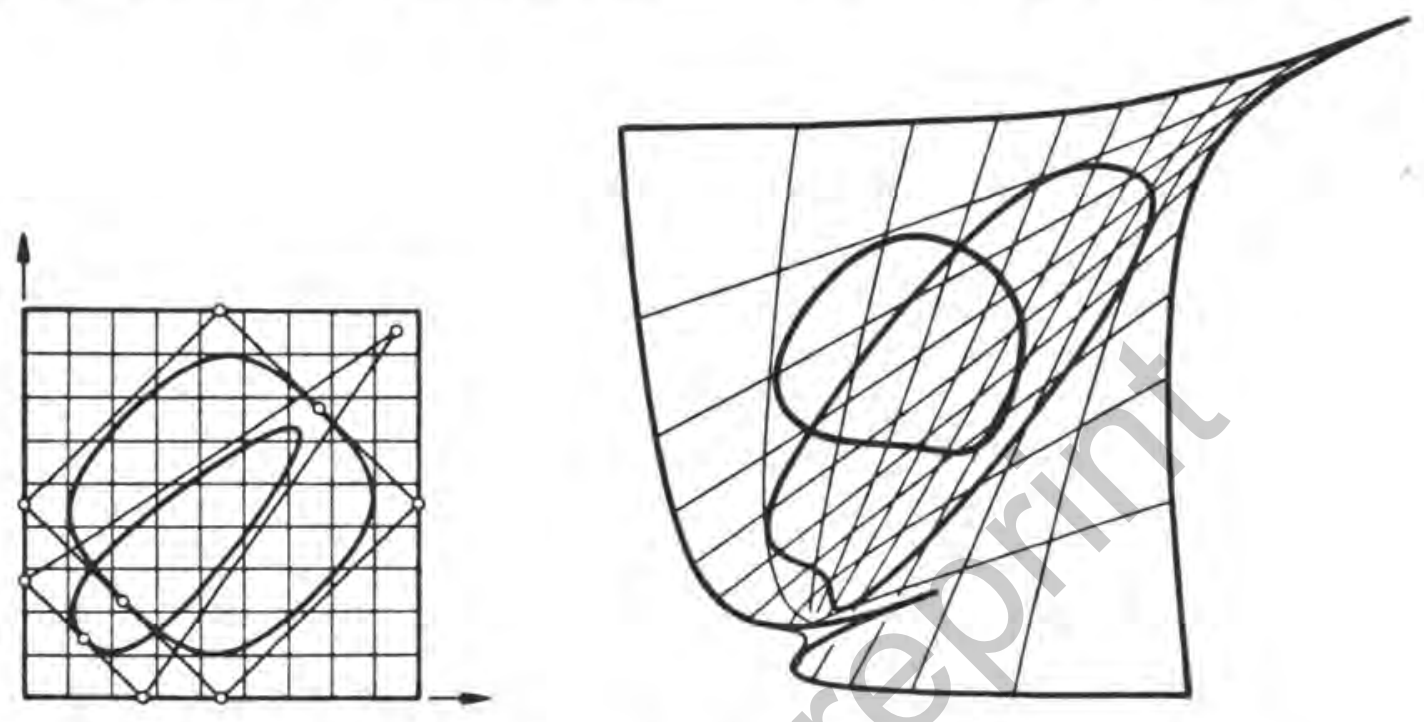

Figure 7. Mapping of a rational quartic and of a closed rational $C^{1}$ cubic on a rational biquadratic TPB-surface
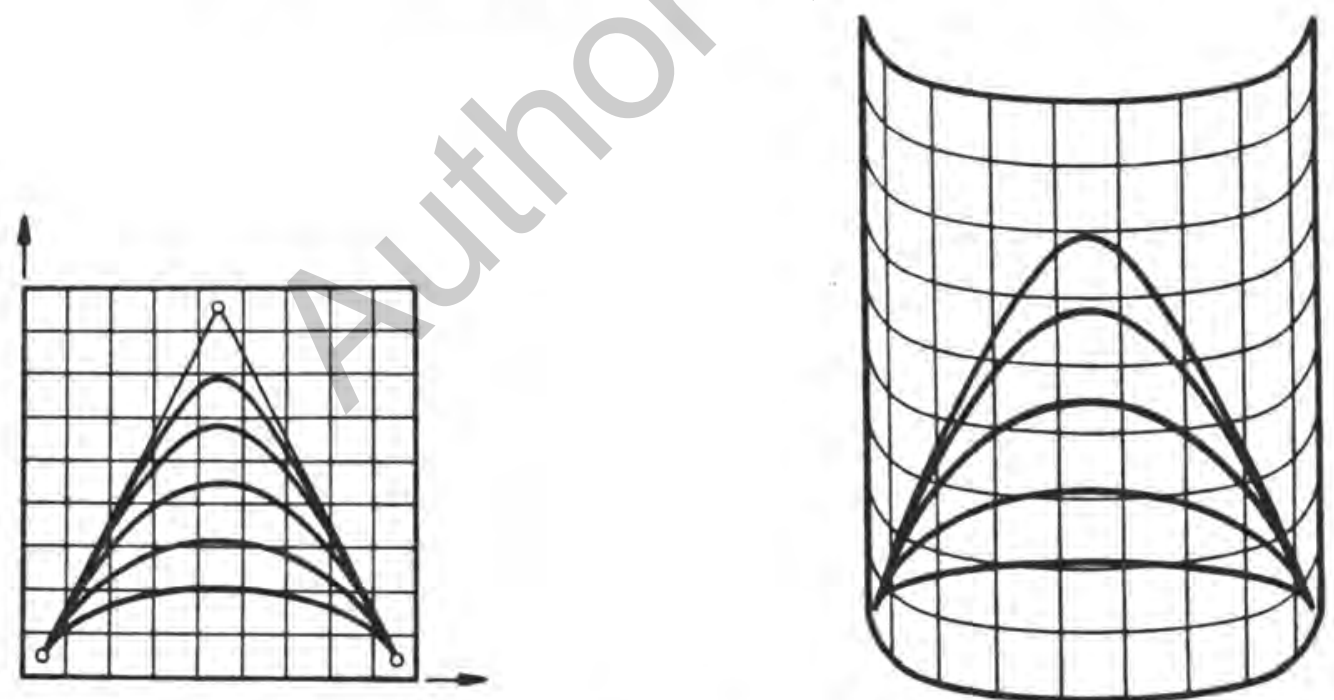

Figure 8. Mapping of rational quadratic curves $\left(\beta_{1}=\frac{1}{4}, \frac{1}{2}, 1,2,4\right)$ on a rational bicubic TPB-surface $\left(\omega_{i, 1}=\omega_{i, 2}=2\right)$

\section{Free Form Deformation}

Theorems 1 and 2 give the exact and explicit description of surface curves. These curves can be thought of as the boundary curves of trimmed surfaces, resulting from intersecting 
or blending operations, for instance. Surface curves described by Theorems I and 2 also can be interpreted as deformations of planar curves under the mapping of the equation which defines the associated free form surface. Therefore, they provide the mathematical foundation for the free form deformation (briefly FFD) approach to planar curve design via surface modelling. ${ }^{2}$

Figure 9 illustrates the example of modelling a planar shape which is bounded by linear and quadratic (Bézier) curves. First, the form is embedded in the domain $[0,1] \times[0,1]$ of a TPB-surface (upper left illustration of Figure 9). The deformation procedure is chosen to be polynomial biquintic, resulting in a corresponding control point net which covers the deformation domain (lower left illustration of Figure 9). The Bézier points of the grid are given by $\mathbf{F}_{i, j}=\left(\frac{i}{5}, \frac{i}{5}, 0\right), 0 \leq i, j \leq 5$. The actual (biquintic) deformation of the form involves the translation of the Bézier points $\mathbf{F}_{i, j} \rightarrow \tilde{\mathbf{F}}_{i, j}$ (right illustration of Figure 9) and evaluation of the FFD defining (biquintic) equation with coefficients $\dot{\mathbf{F}}_{i, j}$. While the position of every point in the interior as well as those on the boundary curves of the object are altered by the deformation equation, Theorem 1 and Statement 1 actually provide an exact and closed form representation of all boundary curves of the form. According to Statement 1, linear isoparametric boundary curves map to quintic Bézier curves, and according to Theorem 1 linear non-isoparametric boundary curves map to Bézier curves of degree 10 while quadratic boundary curves map to Bézier curves of degree 20.
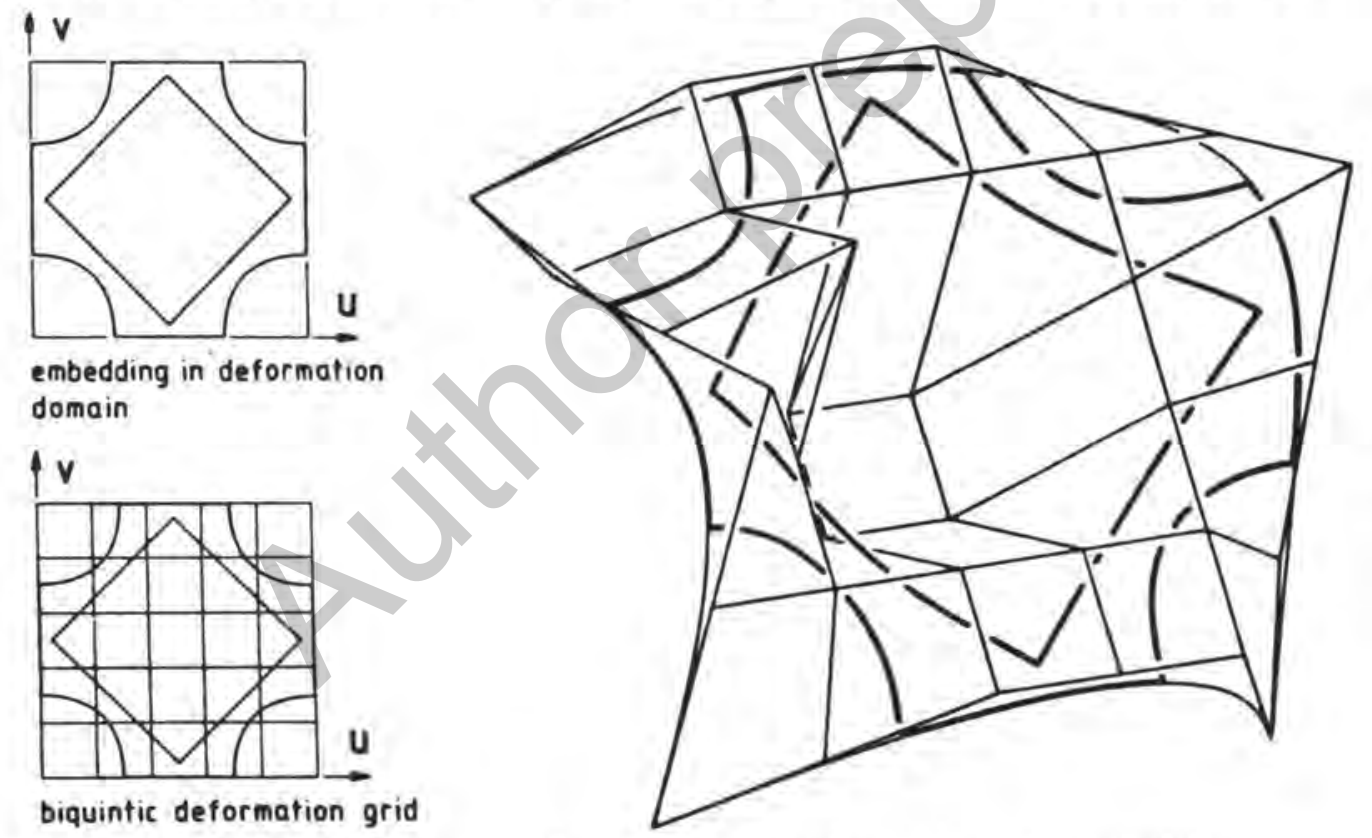

Figure 9. Biquintic deformation of a planar form bounded by linear and quadratic curves

\section{Approximation of Trim Curves}

Major disadvantage of exactly representing trim curves is a rather high degree of $\mathbf{F}(\mathbf{K}(t))$ which might cause problems in following interrogation actions such as intersection oper-

\footnotetext{
${ }^{2}$ An introduction into the FFD idea, i.e. curve and surface modelling by the way of volume design; has been given in [Hos 92].
} 
ations and which also might not be supported by certain CAD systems. Therefore, an approximation of $\mathbf{F}(t)=\mathbf{F}(\mathbf{K}(t))$ by a low polynomial degree Bézier (spline) curve $\mathbf{X}(t)$ might be desirable. An easily implemented quite powerful technique to do so is the one described in [Hos 87] based on parameter optimization and the concept of geometric continuity:

We resumee reduction to cubic (spline) curves only, i.e. we assume the approximating curve $\mathbf{X}(t)$ to be given in Bézier form,

$$
\mathbf{X}(t)=\sum_{k=0}^{3} \mathbf{b}_{k} B_{k}^{3}(t), \quad t \in[0,1],
$$

where $\mathbf{b}_{k}$ are unknown Bézier points of $\mathbf{X}(t)$.

Requiring that the endpoints of $\mathbf{X}(t)$ and of $\mathbf{F}(t)$ coincide, and that the two curves meet each other with first order smoothness at these points, it follows that Bézier points of $\mathbf{X}(t)$ are determined by

$$
\begin{array}{ll}
\mathbf{b}_{0}=\mathbf{B}_{0}, & \mathbf{b}_{1}=\mathbf{B}_{0}+\lambda_{1}\left(\mathbf{B}_{1}-\mathbf{B}_{0}\right) \\
\mathbf{b}_{3}=\mathbf{B}_{r N}, & \mathbf{b}_{2}=\mathbf{B}_{r N}+\lambda_{2}\left(\mathbf{B}_{r N-1}-\mathbf{B}_{r N}\right) .
\end{array}
$$

In order to find the best $\lambda_{1}, \lambda_{2}$ we choose $L+1>r N$ points $\mathbf{P}_{l}$ on $\mathbf{F}(t)$ with respect to the equidistant parameter values $t_{l}=\frac{l}{L}, \mathbf{P}_{l} \equiv \mathbf{F}\left(t_{l}\right), l=0, \ldots, L$, and minimize the absolute value $d=\sum_{l=0}^{L}\left|\mathbf{d}_{l}\right|^{2}$ of the error vectors $\mathbf{d}_{l}=\mathbf{P}_{l}-\mathbf{X}\left(t_{l}\right)$. Substituting (20) in the expression for $\mathrm{d}_{l}$ yields

$$
d=\sum_{l=0}^{L}\left[\mathbf{D}_{l}-\lambda_{1}\left(\mathbf{B}_{1}-\mathbf{B}_{0}\right) B_{1}^{3}\left(t_{l}\right)-\lambda_{2}\left(\mathbf{B}_{r N-1}-\mathbf{B}_{r N}\right) B_{2}^{3}\left(t_{l}\right)\right]^{2},
$$

where

$$
\mathbf{D}_{l} \equiv \mathbf{P}_{l}-\mathbf{B}_{0} B_{0}^{3}\left(t_{l}\right)-\mathbf{B}_{0} B_{1}^{3}\left(t_{l}\right)-\mathbf{B}_{r N} B_{2}^{3}\left(t_{l}\right)-\mathbf{B}_{r N} B_{3}^{3}\left(t_{l}\right) .
$$

$\lambda_{1}$ and $\lambda_{2}$ can be found by solving the linear system of equations resulting from the necessary conditions $\frac{\partial d}{\partial \lambda_{1}}=0$ and $\frac{\partial d}{\partial \lambda_{2}}=0$ for the minimum of $d$.

The result depends on the parametrization of the points $\mathbf{P}_{l}$, of course. In addition, error vectors $d_{l}$ do not necessarily give the shortest distance between points $\mathbf{P}_{l}$ and approximating curve $\mathbf{X}(t)$. Hence, after solving the system we apply a parameter correction $t_{l} \rightarrow t_{i}^{*}$ i.e. $\mathbf{P}_{l} \rightarrow \mathbf{P}_{i}^{*}=\mathbf{F}\left(t_{l}^{*}\right)$, thus, $\mathbf{D}_{l} \rightarrow \mathbf{D}_{i}^{*}, d \rightarrow d^{*}$, as described in [Hos 89] and then solve the new system, which results from $\frac{\partial d^{*}}{\partial \lambda_{1}}=0$ and $\frac{\partial d^{\circ}}{\partial \lambda_{2}}=0$. This process is iterated in order to force nearly all error vectors to be perpendicular to $\mathbf{X}(t)$ in $\mathbf{X}\left(t_{l}\right)$. If the given error tolerance cannot be satisfied, $\mathbf{F}(t)$ is split into two segments at the point where the error is maximal and then the algorithm is applied to each of the two pieces. The algorithm results in a spline curve with a minimal number of cubic Bézier curve segments.

Please note, the Bézier approximation can be built up using the exact point and derivative information of the originally given surface curve in Bézier form, $\mathbf{F}(\mathbf{K}(t))$. At no point of the algorithm estimates of derivative have to be done, neither conversions from or to any other representation have to be performed! 


\section{References}

[Bar 89] L. Bardis, N.M. Patrikalakis: Blending rational B-spline surfaces, EUROGRAPHICS '89 (1989) 453-462.

[Bez 78] P. Bézier: General distortion of an ensemble of biparametric surfaces. Computer-Aided Design 10 (1987) 116-120.

[Cas 87] M.S. Casale : Free-form solid modeling with trimmed surface patches. IEEE Computer Graphics \& Applications 7 (1987) 33-43.

[Cas 89] M.S. Casale, J.E. Bobrow: A set operation algorithm for sculptured solids modeled with trimmed patches. Computer Aided Geometric Design 6 (1989) 235248.

[Cas 92] M.S. Casale, J.E. Bobrow, R. Underwood: Trimmed-patch boundary elements: bridging the gap between solid modeling and engineering analysis. ComputerAided Design 24 (1992) 193-199.

[Cho 89] B.K. Choi, S.Y.Ju: Constant-radius blending in surface modelling. ComputerAided Design 21 (1989) 213-220.

[Cro 87] G.A.Crocker, W.F. Reinke: Boundary evaluation of non-convex primitives to produce parametric trimmed surfaces, SIGGRAPH 87 , ACM Computer Graphics (1987) 129-136.

[DeR 88] T.D. DeRose: Composing Bézier simplices. ACM Transactions on Graphics 7 (1988) 198-221.

[DeR 93] T.D. DeRose, R.N. Goldman, H. Hagen, St. Mann: Functional composition algorithms via blossoming. ACM Transactions on Graphics 12 (1993) 113-135.

[Elb 92] G. Elber: Free form surface analysis using a hybrid of symbolic and numeric computation. PhD Thesis, University of Utah 1992.

[Far 90] G. Farin: Curves and surfaces for computer aided geometric design. A practica] guide. 2. ed. Academic Press 1990.

[Faro 87] R.T. Farouki: Trimmed-surface algorithms for the evaluation and interrogation of solid boundary representations. IBM Journal of Research and Development 31 (1987) 314-334.

[Faro 91] R.T. Farouki: On the stability of transformations between power and Bernstein polynomial forms. Computer Aided Geometric Design 8 (1991) 29-36.

[Fil89] D.J.Filip: Blending parametric surfaces. ACM Transactions on Graphics 8 (1989) 164-173.

[Har 90] T. Harada, H. Toriya, H. Chiyokura: An enhanced rounding operation between curved surfaces in solid modeling. in T.S. Chua, T.L. Kunii (ed.): CG International '90. Springer (1990) 563-588.

[Hos 87] J. Hoschek: Approximate conversion of spline curves. Computer Aided Geometric Design 4 (1987) 59-66.

[Hos 89] J.Hoschek, F.J.Schneider, P. Wassum: Optimal approximate conversion of spline surfaces. Computer Aided Geometric Design 6 (1989) 293-306.

[Hos 90] J. Hoschek, F.J.Schneider: Spline conversion for trimmed rational Bézier- and B-spline surfaces. Computer-Aided Design 22 (1990) 580-590.

[Hos 92] J. Hoschek, D. Lasser: Grundlagen der Geometrischen Datenverarbeitung. 2. ed. Teubner 1992.

[Kla 92] R. Klass, B. Kuhn: Fillet and surface intersections defined by rolling balls. Computer Aided Geometric Design 9 (1992) 185-193. 
[Kob 86] K. Kobori, M. Iwazu, K.M. Jones: Polygonal subdivision of parametric surfaces. in T.L.Kunii (ed.): Advanced computer graphics. Springer (1986) 50-59.

[Kop 91] P.A.Koparkar: Designing parametric blends; surface model and geometric correspondence. The Visual Computer 7 (1991) 39-58.

[Las 91] D. Lasser: Composition of tensor product Bézier representations. Interner Bericht 213/91, Fachbereich Informatik, Universität Kaiserslautern 1991.

[Mil 86] J.R. Miller: Sculptured surfaces in solid models: Issues and alternative approaches. IEEE Computer Graphics \& Aplications 6 (1986) 37-48.

[Nis 90] T. Nishita, Th.W. Sederberg, M. Kakimoto: Ray tracing trimmed rational surface patches. ACM Computer Graphics 24 (1990) 337-345.

[Roc 89] A.P. Rockwood, K. Heaton, T. Davis: Real time rendering of trimmed surfaces. SIGGRAPH '89, ACM Computer Graphics 23 (1989) 107-116.

[Peg 90] J.Pegna, F.E.Wolter: Designing and mapping trimming curves on surfaces using orthogonal projection. Advances in Design Automation I (1990) 235245.

[Sha 88] M. Shantz, S.L. Chang: Rendering trimmed NURBS with adaptive forward differencing. SIGGRAPH '88, ACM Computer Graphics 22 (1988) 189-198.

[She 92] X. Sheng, B.E. Hirsch: Triangulation of trimmed surfaces in parametric space, Computer-Aided Design 24 (1992) 437-444.

[Vri91] A.E. Vries-Baayens: CAD product data exchange: conversions for curves and surfaces. Dissertation, Delft, University Press 1991.

[Vri 92] A.E. Vries-Baayens, C.H. Seebregts: Exact conversion of a trimmed nonrational Bézier surface into composite or basic nonrational Bézier surfaces. in $\mathrm{H}$. Hagen (ed.): Topics in surface modeling. Siam (1992) 115-143 (short version of [Vri 91].

D. Lasser, G.-P. Bonneau

Computer Science

University of Kaiserslautern

67653 Kaiserslautern

Federal Republic of Germany 